Supporting materials for:

\title{
Homogeneously Synchronous Degradation of Chitin into Carbon Dots and Organic Acids in Aqueous Solution
}

\author{
Hui Sü, Jiake Wang ${ }^{\prime}$, Lifeng Yan *
}

CAS Key Laboratory of Soft Matter Chemistry, Hefei National Laboratory for

Physical Sciences at the Microscale, and Department of Chemical Physics, iCHEM, University of Science and Technology of China. Hefei, 230026, China.

$\underline{\text { lfyan@ustc.edu.cn }}$

Number of pages: 4

Number of figures: 3

Number of tables: 1 


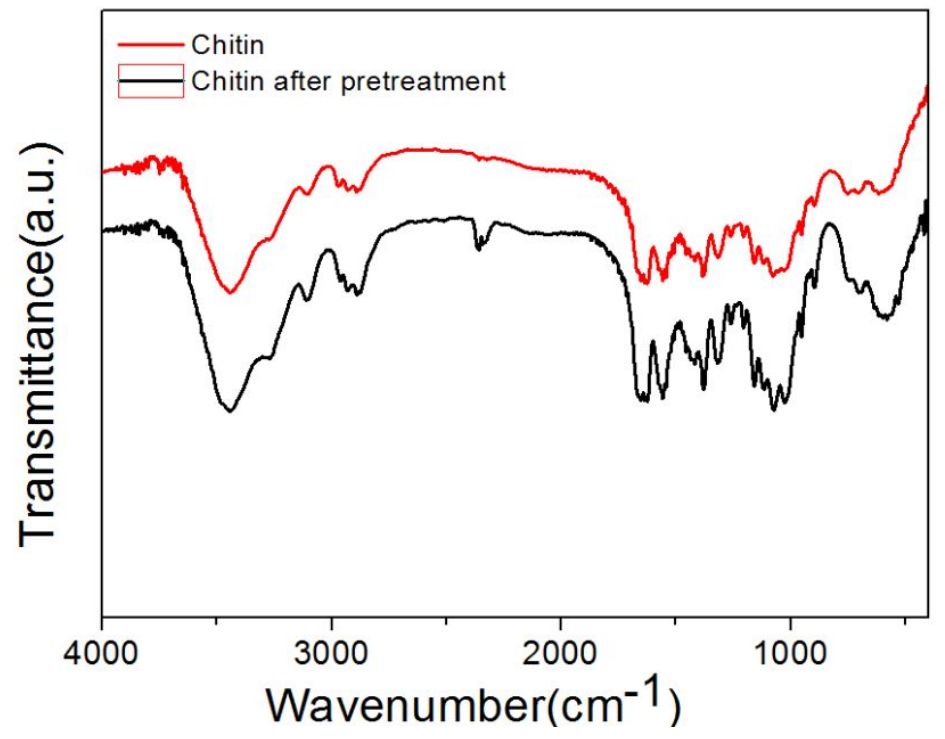

Figure S1. FT-IR spectra of chitin purchased and after pretreatment. 
Table S1. Elemental analysis of chitin purchased and after pretreatment

\begin{tabular}{cccc}
\hline Samples & N (\%) & C (\%) & H (\%) \\
\hline Chitin feedstock & 6.48 & 43.26 & 6.74 \\
\hline After pretreatment & 5.37 & 36.04 & 6.21 \\
\hline
\end{tabular}

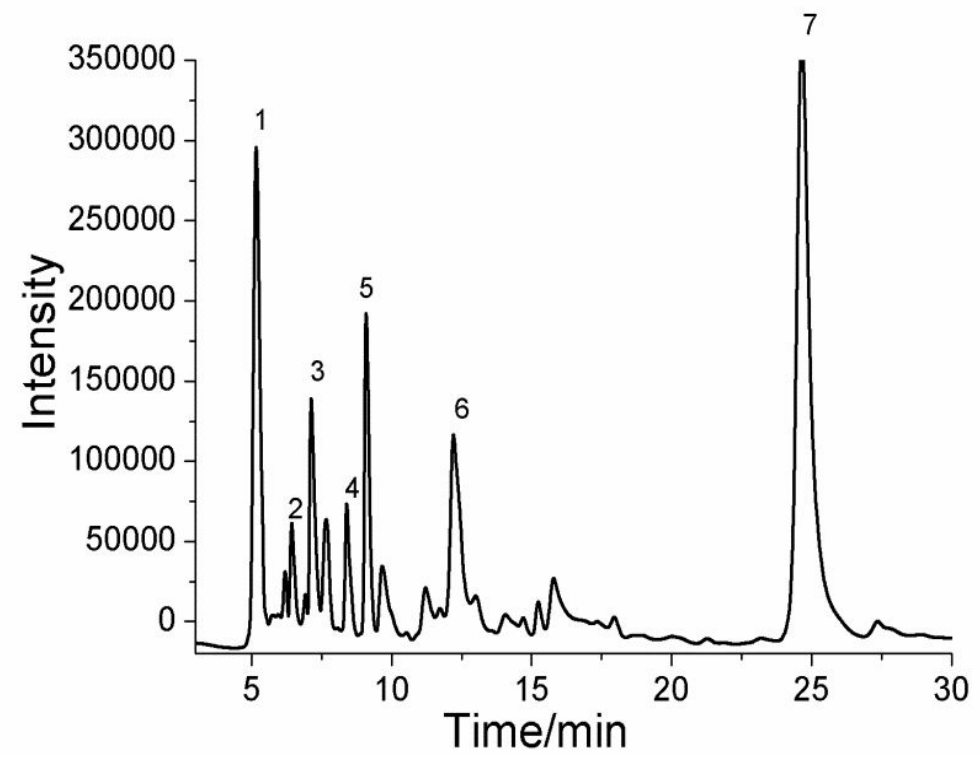

Figure S2. Typical HPLC curve of the products after the degradation of chitin in its homogeneous aqueous solution: 1.oxalic acid, 2.formic acid, 3. malonic acid, 4.lactic acid, 5.acetic acid, 6. fumaric acid, and 7. Pyrrole 

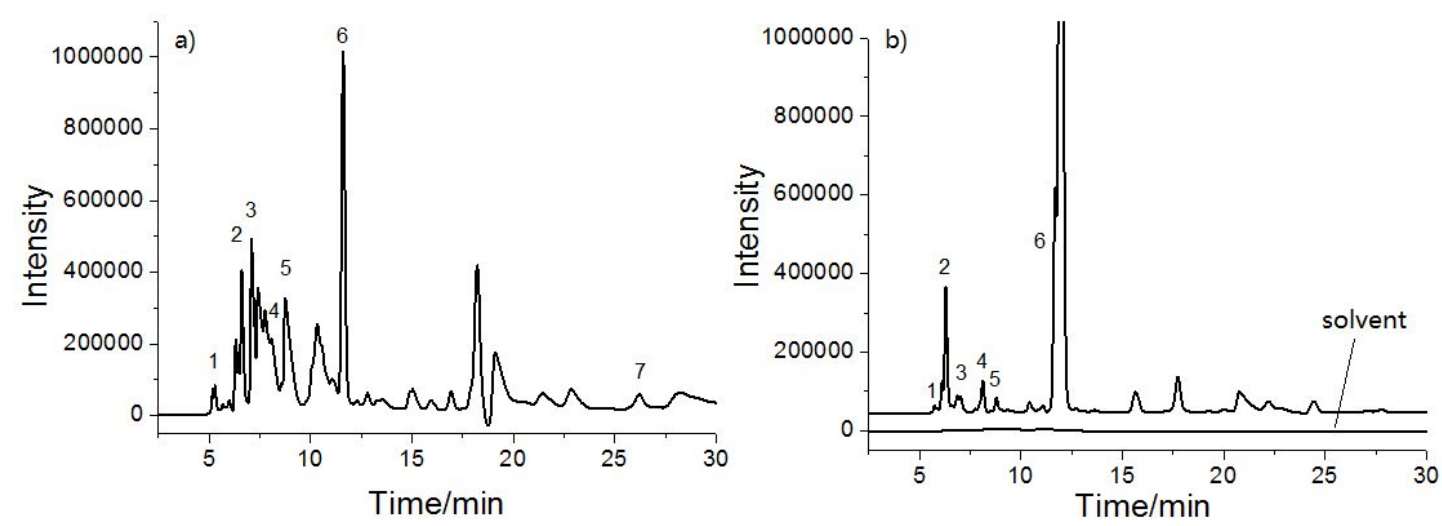

Figure S3. Typical HPLC curves of the products after the hydrothermal degradation of glucosamine (a) and glucose (b) in aqueous solution solvent, solvent curve also showed in (b). 